\title{
Helicobacter pylori-derived extracellular vesicles increased in the gastric juices of gastric adenocarcinoma patients and induced inflammation mainly via specific targeting of gastric epithelial cells
}

\author{
Hyun-Il Choi ${ }^{1}$, Jun-Pyo $\mathrm{Choi}^{2}$, Jiwon $\mathrm{Seo}^{3}$, Beom Jin Kim³ ${ }^{3}$ Mina Rho ${ }^{4}$, Jin Kwan $\mathrm{Han}^{1}$ and Jae Gyu Kim ${ }^{3}$
}

Evidence indicates that Helicobacter pylori is the causative agent of chronic gastritis and perhaps gastric malignancy. Extracellular vesicles (EVs) play an important role in the evolutional process of malignancy due to their genetic material cargo. We aimed to evaluate the clinical significance and biological mechanism of $\boldsymbol{H}$. pylori EVs on the pathogenesis of gastric malignancy. We performed 16S rDNA-based metagenomic analysis of gastric juices either from endoscopic or surgical patients. From each sample of gastric juices, the bacteria and EVs were isolated. We evaluated the role of $\boldsymbol{H}$. pylori EVs on the development of gastric inflammation in vitro and in vivo. IVIS spectrum and confocal microscopy were used to examine the distribution of EVs. The metagenomic analyses of the bacteria and EVs showed that Helicobacter and Streptococcus are the two major bacterial genera, and they were significantly increased in abundance in gastric cancer (GC) patients. H. pylori EVs are spherical and contain CagA and VacA. They can induce the production of tumor necrosis factor- $\alpha$, interleukin (IL)- 6 and IL-1 $\beta$ by macrophages, and IL-8 by gastric epithelial cells. Also, EVs induce the expression of interferon gamma, IL-17 and EV-specific immunoglobulin Gs in vivo in mice. EVs were shown to infiltrate and remain in the mouse stomach for an extended time.

$H$. pylori EVs, which are abundant in the gastric juices of GC patients, can induce inflammation and possibly cancer in the stomach, mainly via the production of inflammatory mediators from gastric epithelial cells after selective uptake by the cells. Experimental \& Molecular Medicine (2017) 49, e330; doi:10.1038/emm.2017.47; published online 12 May 2017

\section{INTRODUCTION}

Gastritis, duodenal ulcers, gastric ulcers and gastric cancer are the most common gastric diseases. The main causes of gastric disease are suggested to be salt, helminth infection, dietary antioxidants and cigarette smoking. Healthy people can also have acute gastritis, which suggests the presence of various other causes. Helicobacter pylori can induce gastric mucosal inflammation and is one of the major causes of peptic ulceration and gastric adenocarcinoma. Approximately half of the world's population is estimated to be colonized by this bacterium. The development of chronic superficial gastritis in normal mucosae from $H$. pylori infection is one of the first steps in the progression to gastric carcinoma. ${ }^{1}$ Over the course of inflammation, interleukin (IL)-8, a chemokine that increases the risk of severe inflammation and gastric cancer in Asian and Caucasian populations, is involved in the innate immune response. However, the details of its role are not yet known. ${ }^{2}$

The gut microbiota, especially in gastric juices, and their gene products affect a host's immune system, development and metabolism. Previously, the sequencing of a shotgun library was conducted to compare adult- and infant-type microbiota ${ }^{3}$ and to characterize microbiota in the human stomach and the effect of $H$. pylori on gastric microbiota. ${ }^{4}$ The introduction of next-generation metagenomic sequencing resulted in an unprecedented understanding of the effect of microbiota in relation to gut health or gut-related diseases. ${ }^{5}$ However, these

${ }^{1}$ Division of Molecular and Life Sciences, Department of Life Science, Pohang University of Science and Technology (POSTECH), Pohang City, Gyeongsangbuk-do, Republic of Korea; ${ }^{2}$ Asan Institute for Life Sciences Asan Medical Center, Seoul, Republic of Korea; ${ }^{3}$ Department of Internal Medicine, Chung-Ang University College of Medicine, Seoul, Republic of Korea and ${ }^{4}$ Department of Computer Science and Engineering, Hanyang University, Seoul, Republic of Korea

Correspondence: Dr M Rho, Department of Computer Science and Engineering, Hanyang University, 222 Wangsimni-ro, Seongdong-gu, Seoul 04763, Republic of Korea.

E-mail: minarho@hanyang.ac.kr

or Professor JG Kim, Department of Internal Medicine, Chung-Ang University College of Medicine, 84 Heukseok-ro, Dongjak-gu, Seoul 06974, Republic of Korea.

E-mail: jgkimd@cau.ac.kr

Received 22 August 2016; revised 23 November 2016; accepted 29 November 2016 
previous metagenomic studies were conducted using genomic DNA isolated from a population of microbial cell pellets obtained from feces ${ }^{3}$ or through gastric mucosal biopsy. ${ }^{4}$ The results showed that Streptococcus was increased but Helicobacter was decreased in the biopsies of gastric cancer patients. ${ }^{6}$ In detecting risk factors of gastric cancer, including gastritis and $H$. pylori infection, gastric juice analysis was a superior alternative to conventional tests, such as a simple endoscopy, especially in the detection of $H$. pylori. ${ }^{7,8}$

Microbial communities inhabiting animal organs produce extracellular vesicles (EVs) that function as intercellular communicators between the microbes and host cells. ${ }^{9}$ EVs are evolutionally conserved, spherically shaped vesicles that exist in a wide range of sizes and are surrounded by a phospholipid bilayer; they not only play a role in removing unwanted waste from inside the cell but also in transmitting genetic information, contributing to the development and normal physiology of cells, which includes immune suppression, antigen presentation, and signal transduction, and the pathophysiology of diverse diseases such as tumor growth, metastasis and angiogenesis. ${ }^{10} \mathrm{EVs}$ are easily detected in culture media and biological fluids and therefore function as excellent biomarkers. Of particular interest are the EV functions related to inflammatory responses. Previous evidence has shown that EVs derived from Escherichia coli, Pseudomonas aeruginosa or Staphylococcus aureus induce pro-inflammatory cytokines and then cause inflammatory diseases such as sepsis, lung inflammation and atopic dermatitis, respectively. ${ }^{1-13}$ In addition, previous reports have shown that $H$. pylori-derived EVs induce apoptosis, and CagA-containing exosomes from $H$. pylori-infected patients induce morphological change in AGS cells. ${ }^{14,15}$

To date, no metagenomic sequencing has been performed in relation to the successive progression of gastric diseases leading to gastric cancer. It is known that $H$. pylori produces EVs, whereas the role of $H$. pylori-derived EVs in the pathogenesis of gastric malignancy remains largely unknown. ${ }^{16,17}$ In the present study, we hypothesized that $H$. pylori-derived EVs are important in the development of gastric inflammation and subsequent adenocarcinoma. To test this, we evaluated the clinical significance of $H$. pylori EVs in gastric juices from gastric cancer patients using metagenomics. We prepared bacterial cell pellets and EVs from gastric juices of healthy controls (HC en) and patients with duodenal ulcer (DU en), gastric ulcer (GU en) or gastric cancer (GC en), which were obtained by endoscopy and from the gastric juices of gastric cancer patients obtained during surgery (GC sg). Genomic DNAs purified from bacterial cell pellets and EVs were then subjected to next-generation metagenomic sequencing of the microbial $16 \mathrm{~S}$ rDNA genes. We compared the changes in microbial taxonomic composition between the populations of bacterial cells and EVs in relation to gastric disease progression. H. pylori-derived EVs were increased in gastric juices from gastric cancer patients compared with healthy controls. Then, we studied the functional mechanisms of $H$. pylori-derived $\mathrm{EVs}$ in vitro and in vivo and verified that $H$. pylori-derived EVs are taken up in stomach epithelial cells by selective targeting.

\section{MATERIALS AND METHODS}

\section{Patients}

All gastric juices were collected between January 2011 and December 2014 from patients who underwent esophagogastroduodenoscopy and gastric surgery at Chung-Ang University Hospital (Seoul, Republic of Korea). All of the samples were gathered from 10 healthy controls and 10 patients each with gastric cancer, gastric ulcers, and duodenal ulcers and from patients undergoing gastric cancer surgery. Esophagogastroduodenoscopy was performed using an Olympus device (GIF-H290 or GIF-H260Z; Olympus, Tokyo, Japan). Then, a washing pipe was used to collect gastric juices from each lesion. Collection of gastric juice through laparoscopic gastrectomy or open gastrectomy was performed using an aseptic process. The medical records of the patients were reviewed with regard to age, sex, medical history, laboratory findings, endoscopic records, including Helicobacter pylori positivity and surgical records. The exclusion criteria were as follows: medication affecting gastric acid secretion, medication with antimicrobials within the previous 4 weeks and gastric surgery. This study protocol was approved by the Institutional Review Board of Chung-Ang University Hospital (IRB No. 10-080-12-23).

\section{$16 S$ rDNA gene-based metagenomic analysis}

DNA extraction and $16 S$ rDNA PCR of bacteria and EVs derived from gastric juices. After sample preparation for EVs, the bacterial portion derived from the gastric juices was extracted using a DNA extraction kit (DNeasy Blood \& Tissue Kit; Qiagen, Hilden, Germany). We followed a standard protocol and confirmed 16S rDNA sequences using PCR. Briefly, for the PCR, we used the universal 16 S primers 27F (5'-GAGTTTGATCMTGGCTCAG- ${ }^{\prime}$ ) and 518R (5'-WTTACCG CGGCTGCTGG-3') at $94^{\circ} \mathrm{C}$ for 3 min followed by 35 cycles of $94^{\circ} \mathrm{C}$ for $15 \mathrm{~s}, 55^{\circ} \mathrm{C}$ for $45 \mathrm{~s}$ and $72^{\circ} \mathrm{C}$ for 1 min with a final elongation step at $72{ }^{\circ} \mathrm{C}$ for $8 \mathrm{~min}$. The PCR products were purified using AMPure beads (Beckman Coulter, Brea, CA, USA).

Library construction and sequencing. The library was prepared using $16 \mathrm{~S}$ rDNA PCR products according to the GS FLX plus library prep guide. The libraries were quantified using a PicoGreen assay (Victor 3, PerkinELMER, MA, USA), and clonal amplification of the purified library was conducted using the GS-FLX plus emPCR Kit (454 Life Sciences, Branford, CT, USA).

Metagenome profiling and analysis. The high-throughput sequencing reads were filtered based on read length $(\geqslant 300 \mathrm{bp})$ and quality score (average Phred score in a window $\geqslant 20$ ). The read counts for each sample are provided in Supplementary Table 2. Operational taxonomy units ( $>97 \%$ similarity) were clustered using the sequence clustering algorithm UCLUST. ${ }^{18}$ Subsequently, taxonomy was assigned to each operational taxonomy unit using QIIME against the $16 \mathrm{~S}$ rDNA sequence database of GreenGenes 8.15.13. ${ }^{19}$ The alpha diversity was measured using the Chaol index. The bacterial composition $(>5 \%$ in multiple samples) at the genus level was plotted in the heatmap, and the statistical significance among the groups was evaluated using the $\mathrm{R}$ package.

\section{Isolation and characterization of $\boldsymbol{H}$. pylori EVs}

Culture of H. pylori in vitro. H. pylori (HP99) was kindly donated by H.C. Jung (Seoul National University, Seoul, Republic of Korea). This strain (HP99) was isolated from a Korean patient and identified as a CagA- and VacA-positive strain. ${ }^{20,21}$ Briefly, H. pylori (HP99) was 
cultured in modified Brain Heart Infusion Broth (Becton, Dickinson and Company, NJ, USA) with $10 \%$ HyClone fetal bovine serum (GE Healthcare Life Sciences, Franklin Lakes, NJ, USA) at $37^{\circ} \mathrm{C}$ with $10 \% \mathrm{CO}_{2}$ to an optical density of 1.2.

EV isolation. The bacteria were cleared by centrifugation at $10,000 \mathrm{~g}$ for $20 \mathrm{~min}$ at $4{ }^{\circ} \mathrm{C}$ and filtered. The filtrated supernatant was concentrated by the tangential flow filtration system (EMD Millipore, Billerica, MA, USA) with a $100-\mathrm{kDa}$ hollow-fiber membrane filter. The concentrate was further cleared through a $0.22-\mu \mathrm{m}$ filter and ultracentrifuged at $150,000 \mathrm{~g}$ for $3 \mathrm{~h}$ at $4{ }^{\circ} \mathrm{C}$. The pellet was resuspended in $1 \mathrm{ml}$ phosphate-buffered saline (PBS). The EVs between a 10 and 30\% OptiPrep solution (Sigma, St Louis, MO, USA) were density-gradient ultracentrifuged at $100,000 \mathrm{~g}$ for $2 \mathrm{~h}$ at $4^{\circ}$ C. Next, PBS was added to the separated EV fractions, and the samples were ultracentrifuged to remove the OptiPrep solution and resuspended in PBS. The final protein concentrates were quantified by the BCA assay (Thermo Scientific, Rockford, IL, USA) and stored at $-80^{\circ}$ $\mathrm{C}$ until they were ready for use.

Transmission electron microscopy. To prepare for transmission electron microscopy analysis, $500 \mathrm{ng}$ of EVs in $10 \mu \mathrm{l}$ of PBS were bound to 300-mesh Cu-coated grids (Electron Microscopy Sciences, Hatfield, PA, USA) and stained with $2 \%$ uranyl acetate. The grid was then incubated for $12 \mathrm{~h}$ until dry and analyzed using a JEM 1011 at $\times 100$ and $\times 200$ resolutions (JEOL, Tokyo, Japan).

Dynamic light scattering. To determine the size distribution profile of the EVs, $1 \mathrm{ml}$ of the EVs $\left(20 \mu \mathrm{g} \mathrm{ml}^{-1}\right)$ was analyzed at $25^{\circ} \mathrm{C}$ using a Zetasizer (Malvern Instruments, Worcestershire, UK) according to the manufacturer's protocols with low-volume disposable sizing cuvettes. There were a total of 30 runs. Three measurements were recorded to verify the consistency.

\section{Functional evaluation of $\boldsymbol{H}$. pylori EVs}

Western blotting. Proteins from H. pylori-derived EVs were separated on a $10 \%$ SDS-polyacrylamide gel electrophoresis and transferred to nitrocellulose membranes. The membranes were blocked with 5\% skim milk and incubated with anti-CagA (sc-25766) and anti-VacA (sc-25790) antibodies (Santa Cruz Biotechnology, Dallas, TX, USA) and secondary antibodies before development.

\section{Analyses of in vitro cytokine production}

Analyses of in vitro cytokine production induced by $H$. pylori-derived EVs. Production of pro-inflammatory cytokines in cultured media from human (differentiated THP-1 cells) and mouse (RAW 264.7 cells) peritoneal macrophage cells and the human stomach epithelial cells (AGS cells) were measured by an enzyme-linked immunosorbent assay (ELISA; R\&D Systems, Minneapolis, MN, USA). Briefly, $5 \times 10^{4}$ macrophage cells and $1 \times 10^{5}$ epithelial cells seeded in 24 -well plates were treated with 10,100 or $1000 \mathrm{ng} \mathrm{ml}^{-1}$ of EVs for $12 \mathrm{~h}$ before the culture media was subjected to analyses.

To confirm the toxicity of the lipopolysaccharide-induced (LPS; E. coli O55: B5; Merck Millipore, Damstadt, Germany) immune response by EVs derived from $H$. pylori, peritoneal macrophages were isolated from wild-type (C57BL/6) and TLR4-deficient mice, and $5 \times 10^{4}$ cells were seeded in a 24 -well plate. The cells were treated with EVs for $12 \mathrm{~h}$ before cytokine levels in culture media were measured by ELISA (R\&D Systems). Six-week-old C57BL/6 mice (The Jackson Laboratories, Bar Harbor, ME, USA) were bred in an animal facility at Pohang University of Science and Technology. H. pylori EVs and bacterium in PBS $(100 \mu \mathrm{g}$ per $100 \mu \mathrm{l})$ were administered intraperitoneally and orally for 4 weeks, respectively. Administration was performed for five consecutive days for the first week and twice a week for the remainder of the schedule (following a protocol in Figure $5 \mathrm{a}$ ). The mice were killed on the 23rd day.

Characterization of T-cell subsets. We seeded $1 \times 10^{6}$ cells from spleens on a 24-well plate coated with $0.1 \mu \mathrm{g} \mathrm{ml}^{-1}$ of anti-CD3 antibodies and anti-CD28 antibodies (eBioscience, San Diego, CA, USA). After incubation at $37^{\circ} \mathrm{C}$ for $12 \mathrm{~h}$, the supernatants were collected, and interferon gamma (IFN- $\gamma$ ), IL-17 and IL-4 cytokine levels were measured in a Versa Max ELISA Microplate Reader (Molecular Devices, Sunnyvale, CA, USA).

Quantitation of serum antibodies. The serum immunoglobulin (Ig) $\mathrm{G}_{1}$ antibody level was measured by ELISA (Bethyl Laboratories, Montgomery, TX, USA) for $\operatorname{IgG}_{1} \mathrm{~s}$ specific for EVs according to the suggested protocols.

Fluorescent imaging of EVs in vitro and in vivo. H. pylori and H. pylori-derived EVs were labeled with Cy7 (Cy7 Mono NHS Ester, GE Healthcare Bio-Sciences, Pittsburgh, PA) in PBS for $1 \mathrm{~h}$ at room temperature and were orally administered to abdomen-shaved C57BL/6 mice. The fluorescence was measured using an IVIS spectrum CT (PerkinElmer, Waltham, MA, USA) at 780-800 nm wavelengths. EVs labeled with DiI Stain (1,1'-dioctadecyl-3,3,3', 3'-tetramethylindocarbocyanine perchlorate ('DiI'; DiIC18(3), Thermo Scientific)) were applied to $1 \times 10^{5}$ epithelial cells seeded on gelatin-coated glass for $12 \mathrm{~h}$. The cells were washed with PBS, fixed with $4 \%$ paraformaldehyde in $\mathrm{PBS}$ at $37^{\circ} \mathrm{C}$ for $1 \mathrm{~h}$, and stained with DAPI (Invitrogen, Waltham, MA, USA) for $10 \mathrm{~min}$.

\section{Statistical analysis}

All of the statistically significant differences were calculated using GraphPad Prism 5.0 (GraphPad Software, San Diego, CA, USA). A $t$-test was used for single comparisons, and one-way analysis of variance was used for multiple comparisons. The statistical significance was set at $P<0.05$.

\section{RESULTS}

\section{Characterization of EVs derived from the gastric juices of patients with gastric disease}

EVs were purified from gastric juices collected from patients with duodenal ulcer ( $\mathrm{Du}$ en), gastric ulcers (GU en) or gastric cancer (GC en) and from healthy controls (HC en). Transmission electron microscopy images showed that the EVs were spherical in shape regardless of the disease type (Figure 1a). A dynamic light scattering analysis showed that the size of EVs was heterogeneous with diameters ranging from 20 to $200 \mathrm{~nm}$ (Figure $1 \mathrm{~b}$ ).

$16 \mathrm{~S}$ rDNA gene-based metagenomic analyses of bacteria and EVs from endoscopically obtained gastric juices from $\mathrm{HC}$ and patients with DU, GU or GC

$16 \mathrm{~S}$ rDNA was sequenced from purified DNA collected from bacteria-containing pellets and EVs in gastric juices of $\mathrm{HC}$ and patients with DU, GU or GC. Alpha diversities (withincommunity diversities) using Chaol indices were measured to estimate species richness in each group (HC, DU, GU and GC) based on $16 \mathrm{~S}$ rDNA gene sequencing data for bacterial 
a

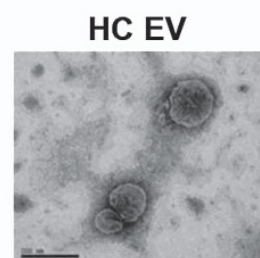

\section{GU EV}

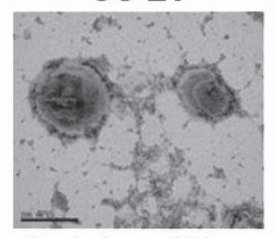

Scale bar $-200 \mathrm{~nm}$
DU EV

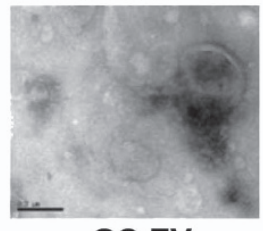

GC EV

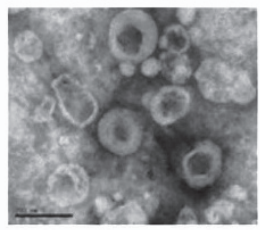

Bacteria

C

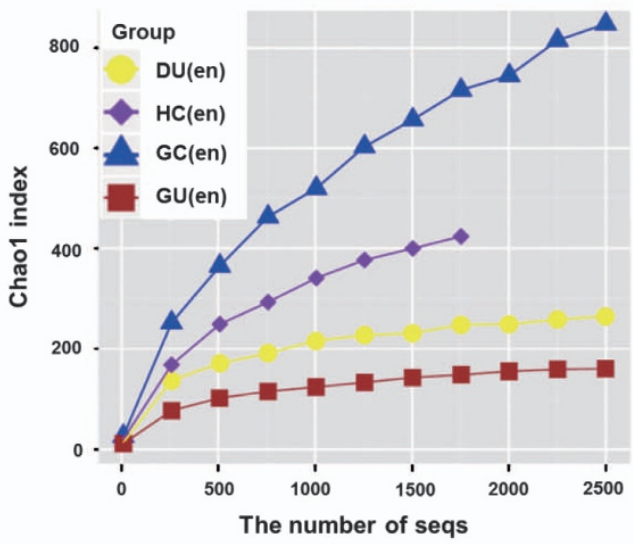

b
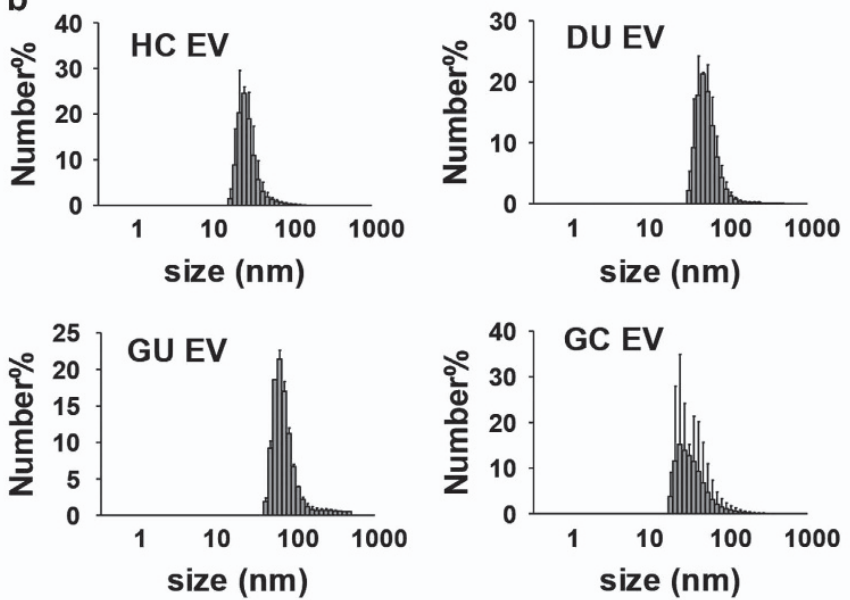

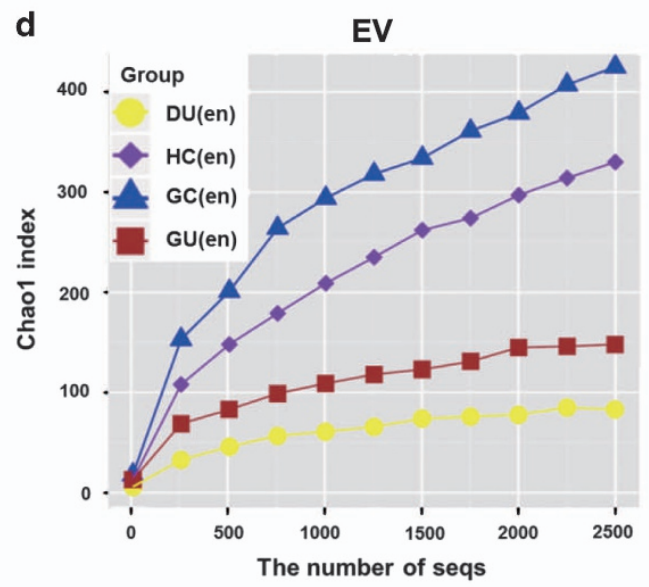

e
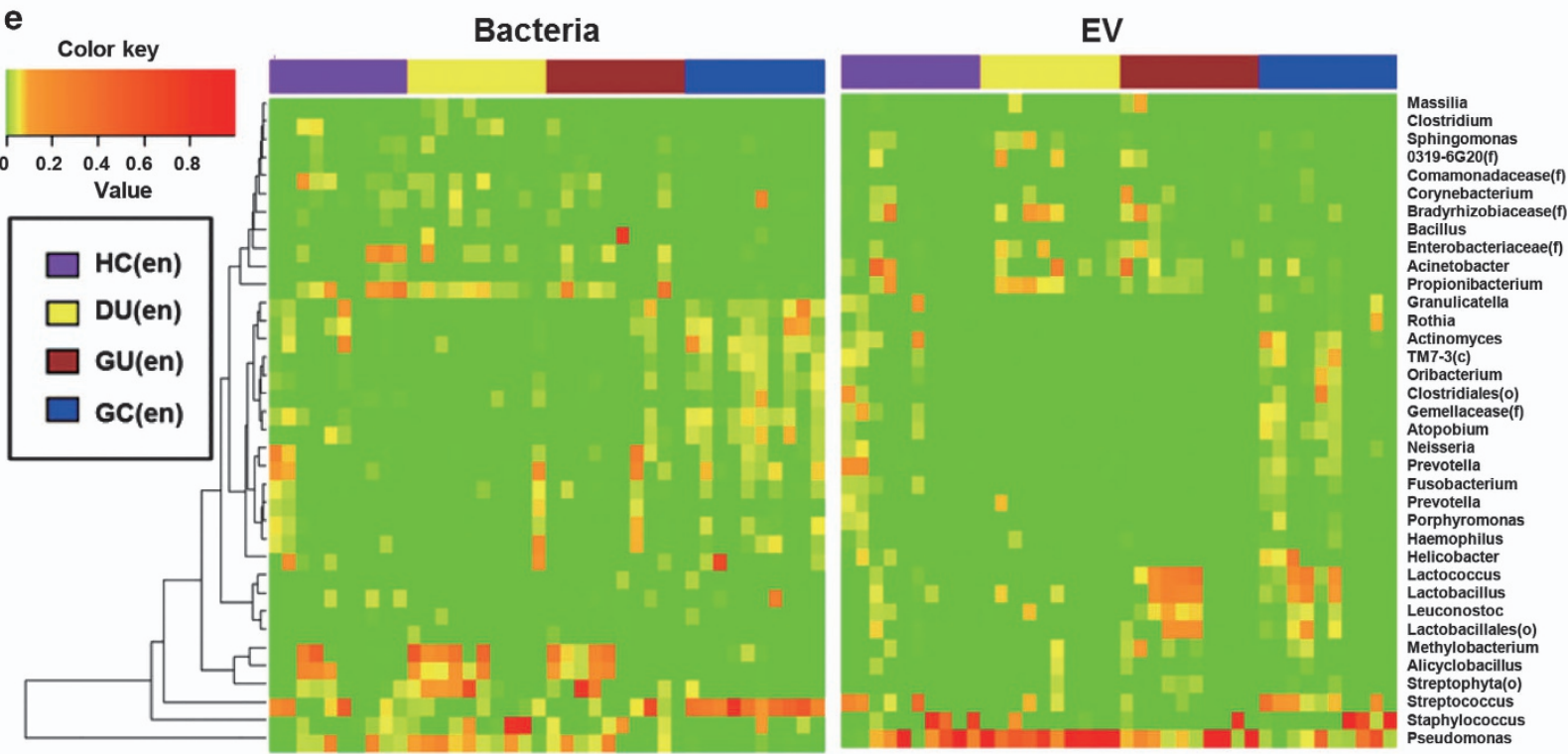

Prevotella are two of the OTU will have a very different sequence (sequence similarity of $60 \%$ ).

Figure 1 Characterization of extracellular vesicles (EVs) and the composition profiling derived from the gastric juices of patients. (a) Transmission electron microscopy (TEM) images of EVs derived from gastric juices. (b) Size distribution of EVs determined using dynamic light scattering (DLS). (c) Alpha diversity of the Genome Sequencer FLX+ system-based sequencing of 16S rDNA genes in bacteria samples. (d) Alpha diversity of the Genome Sequencer FLX+ system-based sequencing of 16S rDNA genes in EVs. (e) Heatmap comparing the relative abundance of bacteria and EVs at the genus level in gastric juices from patients. 
pellets and EVs, respectively. The GC group had the highest Chaol index and was the most diverse among the four groups for both the bacterial pellets and EVs, followed by the HC group. The GU group was the least diverse among the four groups in its bacterial composition, whereas the DU group was the least diverse based on the EV composition (Figures 1c and d). Common microorganisms among the four groups were selected based on the relative abundance in each sample and are shown as a heatmap (Figure 1e). Streptococcus $(P<0.05)$, Gemellaceae $(P<0.05)$, Oribacterium $(P<0.05)$ and TM7-3 $(P<0.01)$ were increased in the bacterial pellets and EVs from gastric cancer (GC en) patients compared to the healthy control (HC en) patients. Although not statistically significant, there was an increase in Helicobacter in EVs and bacterial pellets (Supplementary Figures 1 and 2 and Supplementary Table 1).

$16 \mathrm{~S}$ rDNA gene-based metagenomic analyses of the bacteria and EVs from surgically obtained gastric juices from GC patients

We validated the results of the $16 \mathrm{~S}$ rDNA gene-based metagenomic analysis from the endoscopically obtained gastric juices of GC patients using the sequencing of gastric juices that were directly collected during surgery. For both the bacterial pellets and EVs, gastric cancer samples obtained by endoscopy (GC en) and surgery (GC sg) showed almost identical patterns of alpha diversity, which was significantly higher than the diversity observed in the $\mathrm{HC}$ en specimens (Figure 2a). At a genus level, several microorganisms showed differential abundance among the healthy control ( $\mathrm{HC}$ en), endoscopic (en) gastric cancer and surgical (sg) gastric cancer samples in both bacterial pellets and EV samples (Figure 2b). Common microorganisms among the three groups were selected, and their relative abundance in each sample is shown as a heatmap (Figure 2c). Based on this, we compared healthy controls (en) to gastric cancer (en) and gastric cancer (sg) samples for the bacterial pellets and EVs (Table 1).

Difference in 16S rDNA gene-based metagenomes of the bacteria and EVs from surgically obtained gastric juices from GC patients

Some bacteria were increased in the cancer group. In recent reports, biopsies of gastric cancer patients showed an increase in Streptococcus but a decrease in Helicobacter. ${ }^{4,22,23}$ Therefore, we focused on Helicobacter. In particular, high differential abundance was observed for Helicobacter. A higher level of Helicobacter in the bacterial samples from the surgical gastric cancer group was observed in comparison to the healthy control group $(P=0.06)$ (Figure 3a). In particular, EVs showed a statistically higher level of Helicobacter in the surgical gastric cancer (sg) group than in the healthy control group $(P<0.05$; Figure $3 \mathrm{~b})$. Patient-wise, Helicobacter showed a strong correlative increase between bacterial cells and EVs. These results suggest that even though multiple microorganisms showed differential abundances among stomach-related diseases, only Helicobacter showed significant and correlated changes in regard to gastric diseases in both bacterial cells and EVs (Figure 3c).

\section{Characterization of $\boldsymbol{H}$. pylori-derived EVs}

To characterize the physical and biochemical properties of H. pylori-derived EVs free of bacteria, the purified EVs were observed by transmission electron microscopy. Spherical EVs (arrows, Figure 4a) with a diameter range of $25-200 \mathrm{~nm}$ were observed (Figure $4 \mathrm{~b}$ ).

To test if EVs contain $H$. pylori-specific virulence factors, Western blots of $H$. pylori extracts and EVs were probed with toxin-specific antibodies (Figure 4c). The extracts of wild-type H. pylori showed the presence of CagA and VacA proteins with higher amounts of VacA than CagA ('Hp,' Figure 4c). This detection is specific because the extract from the CagA knockout strain of $H$. pylori $(\mathrm{Hp} \Delta \operatorname{CagA})$ showed a complete absence of CagA protein, whereas VacA remained constant (Figure 4c). Both CagA and VacA were present in H. pylori-derived EVs (lane 1, Figure 4c), and their relative abundance resembled the pattern observed in $H$. pylori cells. This shows that cell-free EVs that contain CagA and VacA virulence factors can be purified from $H$. pylori.

In vitro production of pro-inflammatory cytokines induced by $H$. pylori-derived EVs in human stomach epithelial cells Previous evidence has shown that $H$. pylori induces pro-inflammatory cytokines, such as tumor necrosis factor- $\alpha$ (TNF- $\alpha$ ), IL-1, IL-6 and IL-8, through Toll-like receptor (TLR) signaling pathways. ${ }^{24-26}$ We evaluated whether $H$. pyloriderived EVs containing virulence factors could induce the same group of cytokines. We used human stomach epithelial cells (AGS) that did not have inflammatory cytokines induced by LPS but in which IL-1 $\beta$ is known to activate IL- 8 synthesis. $^{27,28}$ The treatment of AGS with $H$. pylori-derived EVs also induced IL-8 in a dose-dependent manner (Figure 4d). These results show that $H$. pylori-derived EVs induce cytokines independently of LPS.

In vitro production of pro-inflammatory cytokines from macrophage cells of mice induced by $\mathrm{H}$. pylori-derived EVs We next tested whether $H$. pylori-derived EVs can simulate the activity of LPS in inducing pro-inflammatory cytokines in vitro. Peritoneal macrophages from C67BL6/J mice that were treated with LPS showed a drastic increase in IL-6 in culture media; treatment of cells with $H$. pylori-derived EVs also induced dose-dependent IL-6 induction (Figure 5a). Induction of IL-6 by LPS was completely suppressed in a TLR4 knockout strain of C67BL6/J. However, TLR4 knockout did not abolish the ability of $H$. pylori-derived EVs to induce IL-6. Levels of induced cytokines of macrophages from the wild-type C67BL6/J were higher than those of the TLR4 knockout (Figure 5b). Therefore, LPS was also important for inducing inflammatory cytokines. 
a

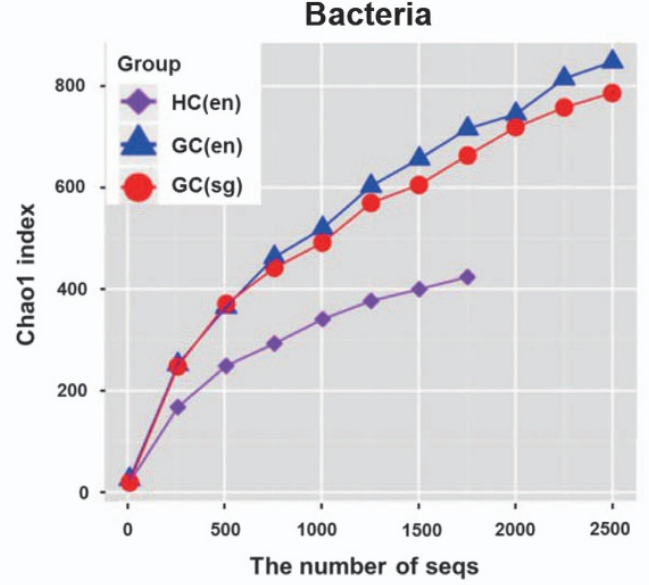

C

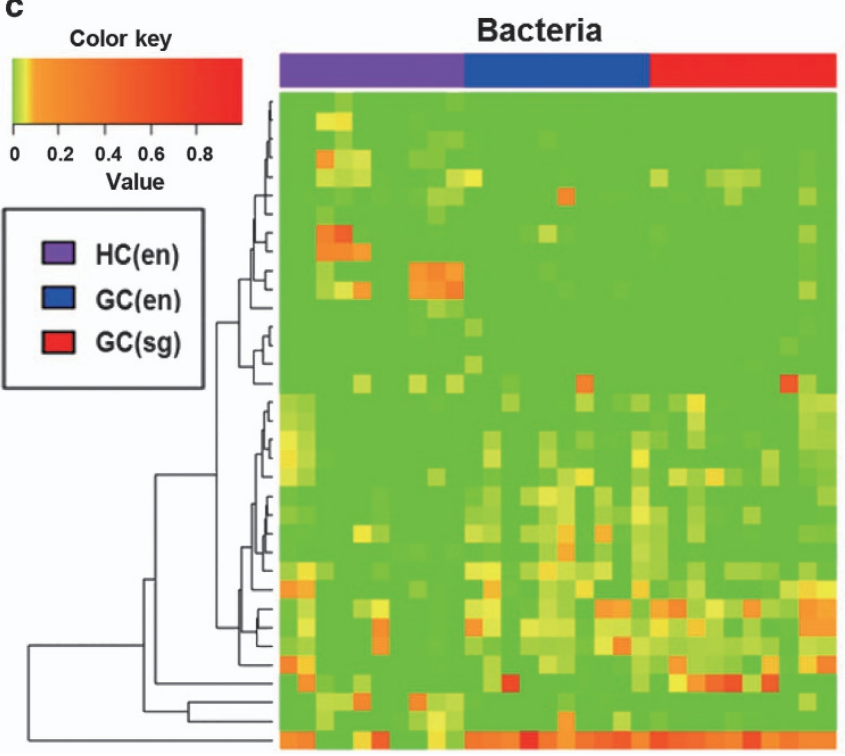

b

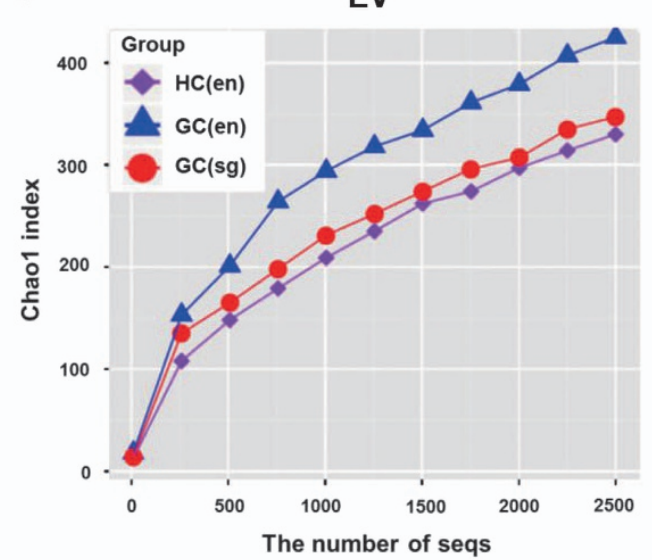

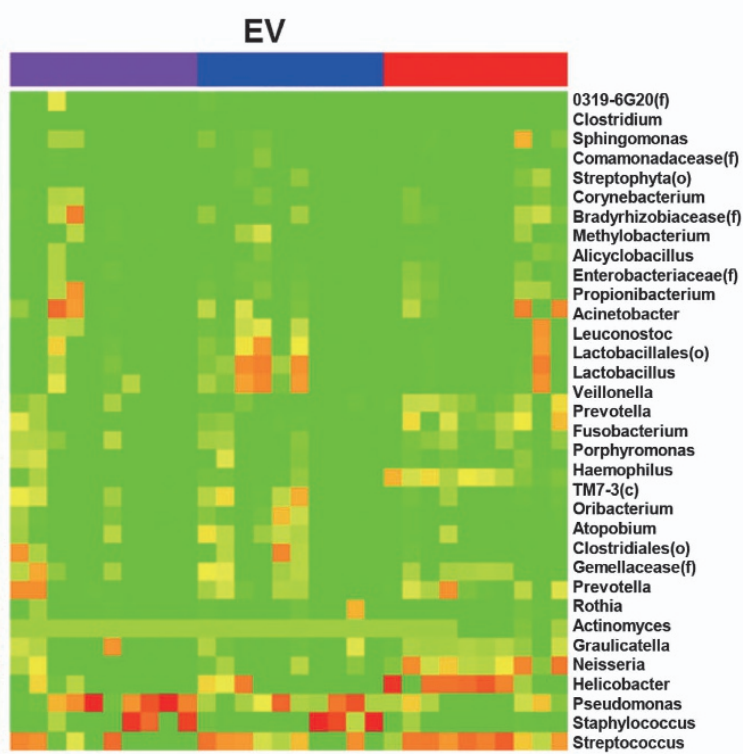

Figure 2 Composition profiling derived from gastric juices obtained directly during surgery. (a) Alpha diversity of the Genome Sequencer FLX+ system-based sequencing of 16S rDNA genes in bacteria samples. (b) Alpha diversity of the Genome Sequencer FLX+ system-based sequencing of $16 \mathrm{~S}$ rDNA genes in extracellular vesicles (EVs). (c) Heatmap comparison of the relative abundance of bacteria and EVs at the genus level in gastric juices from patients.

\section{Characterization of adaptive immune responses induced by cell-free EVs}

Because we showed that cell-free EVs from H. pylori could induce innate immune network-mediated inflammatory responses in vitro and in vivo, we next determined the effects of cell-free EVs on adaptive immune responses (Figure $5 \mathrm{c}$ ). Extracted spleens were tested for IFN- $\gamma$ and IL-17, and representative cytokines for $\mathrm{TH}_{1}$ and $\mathrm{TH}_{17}$-mediated adaptive immune responses, respectively. IFN- $\gamma$ was significantly induced in the spleens from mice that received $H$. pylori bacteria orally compared with the blank media treatment, and cell-free EVs also induced to $\sim 50 \%$ of the level that was induced by bacterial administration (Figure 5d, 1st graph). Intraperitoneal administration of $H$. pylori induced even higher levels of IFN- $\gamma$ and IL-17 than when the bacteria were administered orally (Figure 5d, 2nd graph). Equivalent levels of IL-4 were induced in mice with oral or intraperitoneal administration, and bacteria and cell-free EVs from $H$. pylori induced equivalent levels of IL-4 (Figure 5d, 3rd graph). Next, the generation of immunoglobulin was measured to determine if cell-free EVs could induce sufficient and specific antibody generation. EV-specific $\operatorname{IgG}_{1}$ was generated by oral administration of bacteria and EVs, and the level of $\mathrm{IgG}_{1}$ generation was highly increased by intraperitoneal administration of both bacteria and EVs (Figure 5e). These data indicate that cell-free EVs from $H$. pylori can induce $\mathrm{TH}_{1}$ - and $\mathrm{TH}_{17}$-mediated adaptive immune responses in vivo to a level similar to that induced by bacterial infection, and oral administration of $H$. pylori-derived EVs showed a more localized immune response compared with intraperitoneal administration.

\section{Absorption and distribution of $\mathrm{H}$. pylori and their secreting EVs after oral ingestion in mice}

To examine the kinetics of a whole-body distribution of cell-free EVs, Cy7-labeled H. pylori and cell-free EVs were 
Table 1 Abundance and classification of bacteria and EVs from gastric juices of GC patients.

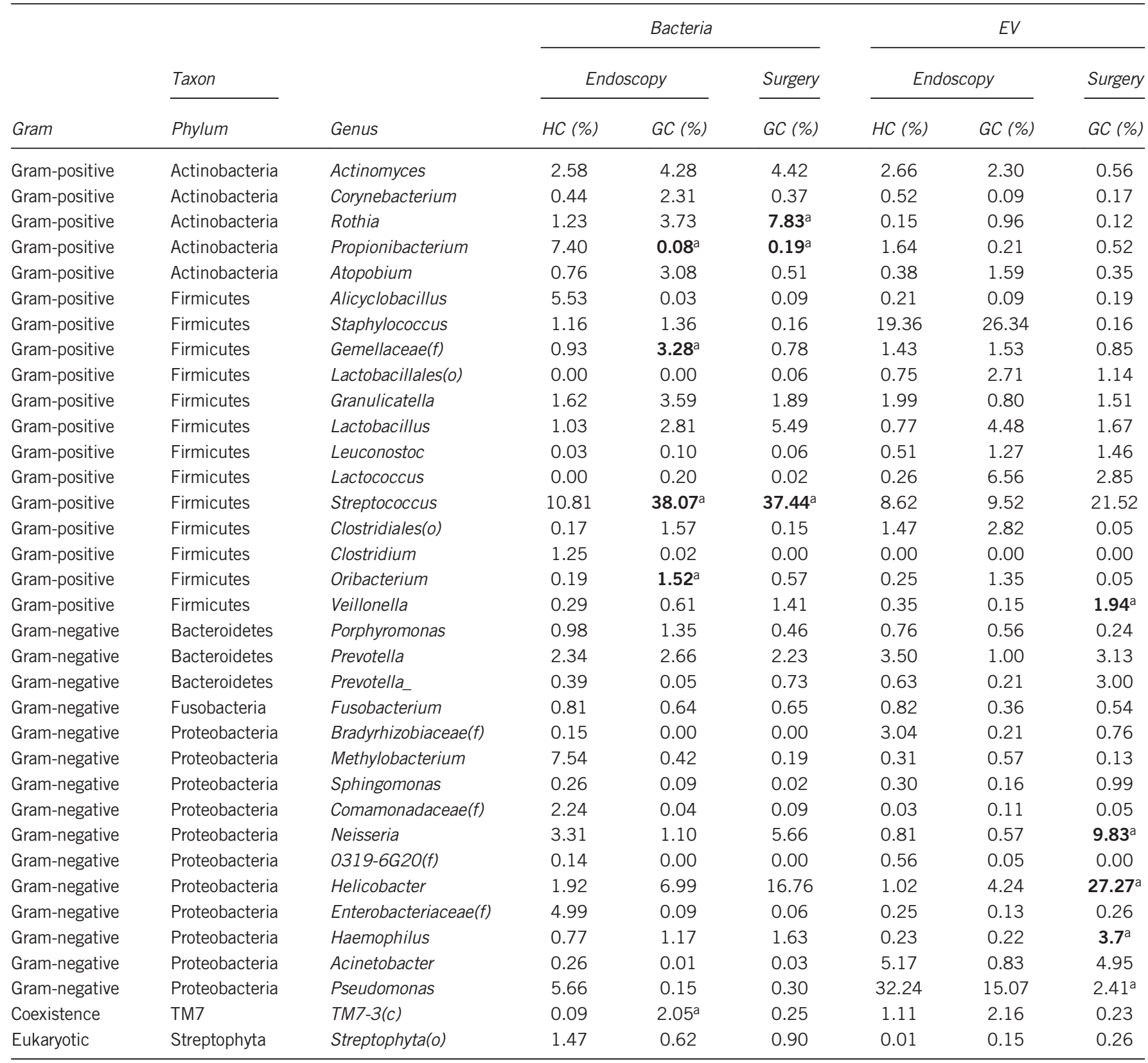

Abbreviations: GC, gastric cancer group; HC, healthy control.

For all the groups $n=10$. The mean value (\%) is provided. These results are comparisons between $\mathrm{HC}$ and other groups.

a $P$-values $<0.05$ are shown in bold.

orally administered to C57bL/6 mice, and the fluorescence was measured using the IVIS spectrum CT (based on a previously published protocol). ${ }^{29}$ Oral administrations of both bacteria and cell-free EVs resulted in strong Cy7 signals in the mouths and stomachs of mice, whereas PBS administration did not produce any Cy7 signals. Strong Cy7 signals were observed as soon as the bacteria and EVs were ingested and remained up to $3 \mathrm{~h}$ after administration. Cell-free EVs remained in the stomach area for up to $24 \mathrm{~h}$ after EV administration, whereas bacterial signals were absent at the 24-h time point (Figure 6a).
Examination of mouse tissues $24 \mathrm{~h}$ after the administration revealed that strong Cy7 signals remained only in the stomach when Cy7-labeled EVs were administered. In comparison, administration of PBS and bacteria did not show any signals in any of the tissues $24 \mathrm{~h}$ after ingestion (Figure 6b). Quantitation of Cy7 fluorescence confirmed the presence of residual EVs in the stomach (Figure 6c) and consequently demonstrated that the penetrating ability of EVs was higher than that of bacteria. Collectively, these findings suggest that H. pylori EVs specifically target stomach epithelial cells. 
a

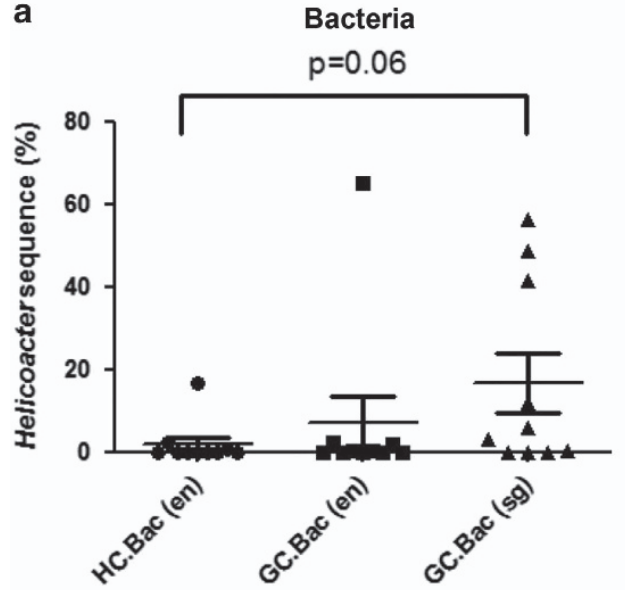

b

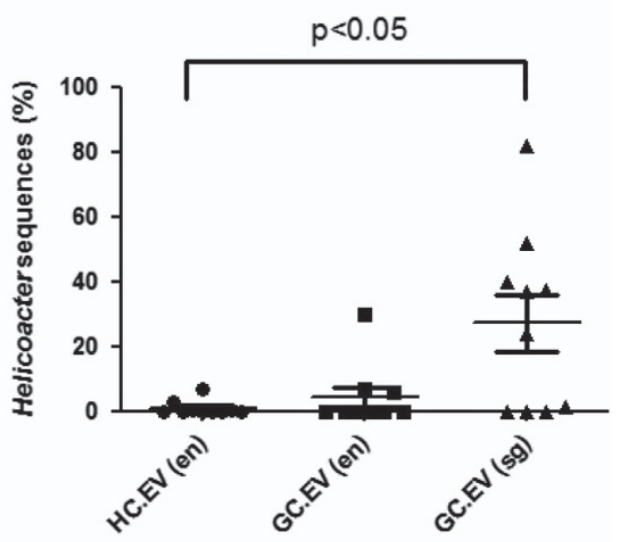

C

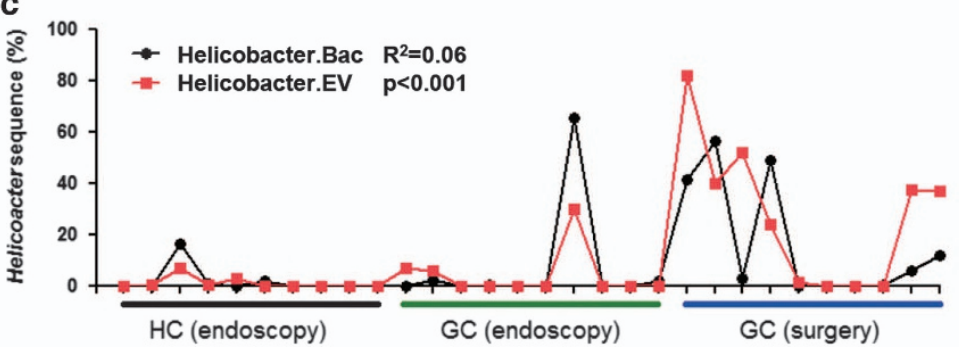

Figure 3 Comparison and correlation between groups of Helicobacter. (a) Percentage of Helicobacter-specific sequence reads obtained by analyzing bacterial pellets and extracellular vesicles (EVs) from gastric juices. (b) Percentage of Helicobacter-specific sequence reads obtained by analyzing bacterial pellets and EVs from gastric juices. (c) Correlation between bacteria and EVs from gastric juices.

a

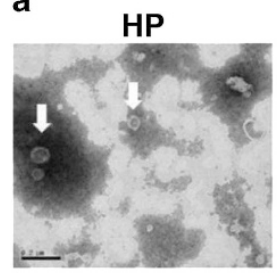

Scale bar $-200 \mathrm{~nm}$
HP EV

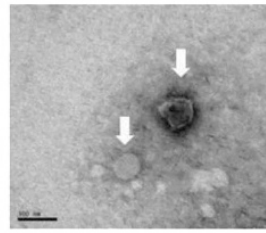

C

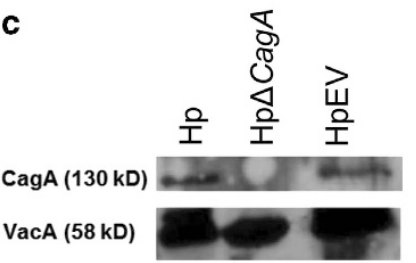

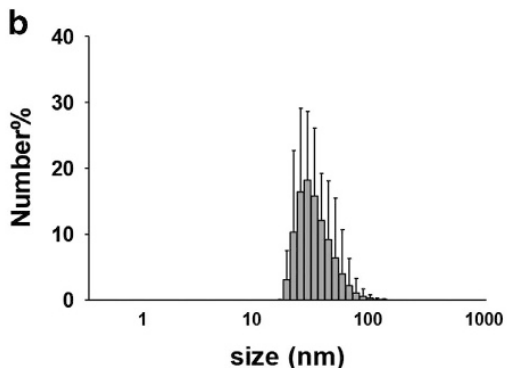

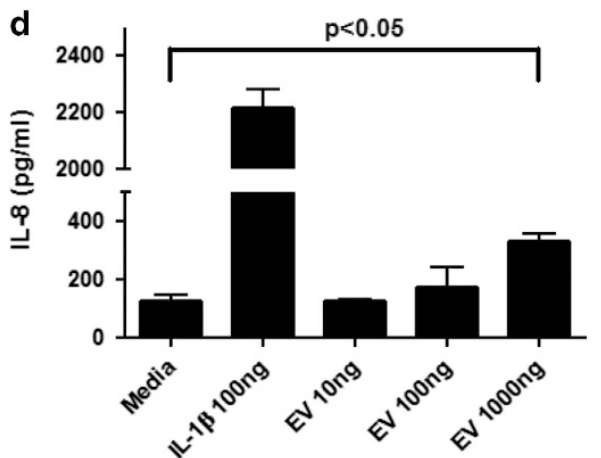

Figure 4 Characterization of purified H. pylori-derived extracellular vesicles (EVs) and induction of IL-8 with EVs in vitro. (a) Transmission electron microscopy (TEM) images. (b) Size distribution of EVs determined using dynamic light scattering (DLS). (c) Confirmation of CagA and VacA in cell extracts and EVs by western blotting. Hp, Hp $\Delta$ CagA and HpEV denote cell extracts from wild-type $H$. pylori strain HP99 $(\mathrm{Hp})$, isogenic H. pylori with CagA gene deletion ( $\mathrm{Hp} \Delta$ CagA), and EVs from $H$. pylori strain HP99 (EV), respectively. (d) Induction of IL-8 in human gastric adenocarcinoma cell line AGS by IL-1 $\beta$ (positive control) and EVs $\left({ }^{*} P<0.05\right.$ refers to a significant difference compared with the phosphate-buffered saline treatment). 
a

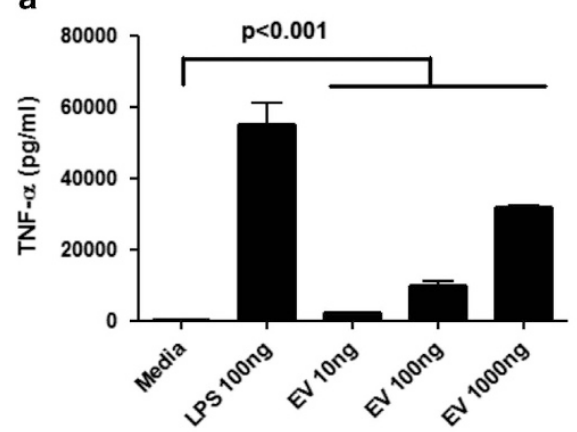

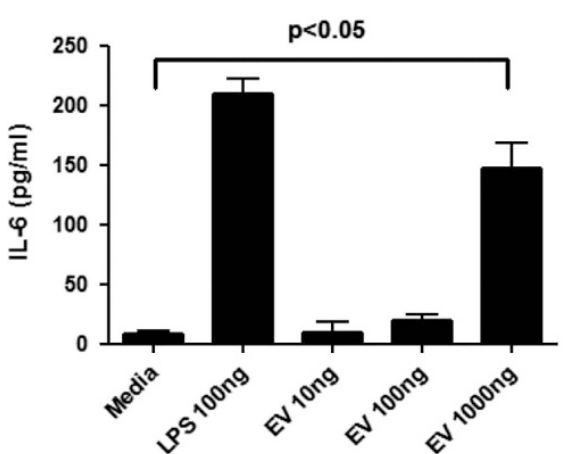

C

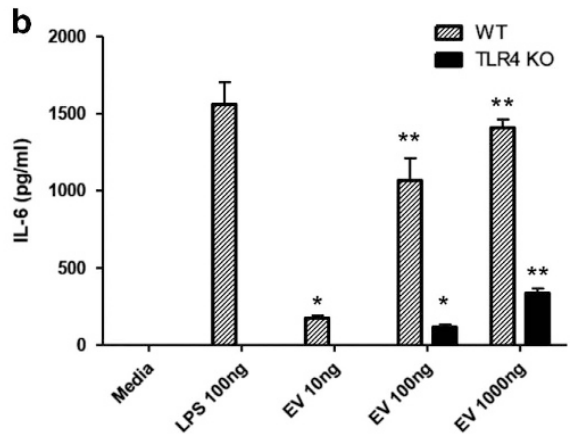

Hp EV $(100 \mu \mathrm{g})$ P.O \& I.P

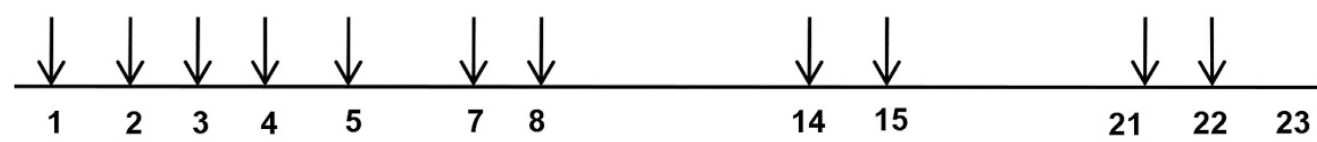

Day

$\begin{array}{lll}1 & 3 & 4\end{array}$

78

15

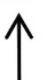

Evaluation

d
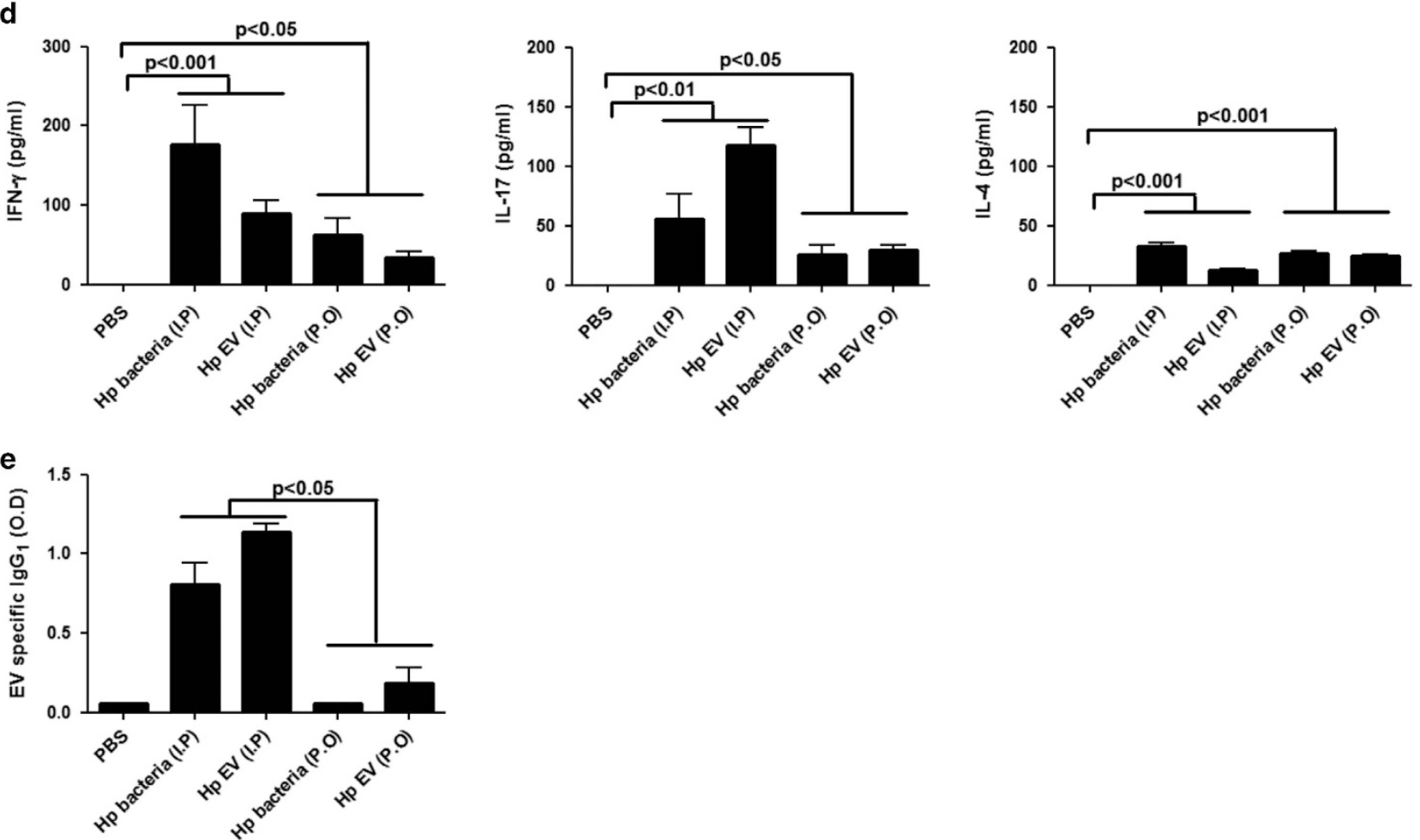

Figure 5 Extracellular vesicle (EV)-induced $\mathrm{TH}_{1}$ and $\mathrm{TH}_{17}$ adaptive immune responses in vivo. (a) Induction of $\mathrm{TNF}-\alpha$ and IL-6 in mouse macrophage RAW 264.7 cells by lipopolysaccharide (LPS) and EVs. (b) Induction in vitro of interleukin (IL)-6 in peritoneal macrophages from C67BL6/J mice following LPS or EV administration (gray bar), and corresponding responses from peritoneal macrophages from the TLR4 knockout (KO) C67BL6/J mice (black bar) $\left({ }^{*} P<0.05,{ }^{*} P<0.01\right.$ refer to significant differences compared with the phosphatebuffered saline (PBS) treatment). (c) A protocol for the oral and intraperitoneal administration of $H$. pylori and $H$. pylori-derived EVs in C57BL/6 mice. (d) Expression levels of $\mathrm{TH}_{1}$ and $\mathrm{TH}_{17}$ type cytokines IFN- $\gamma$, IL-17 and IL-4 measured from the culture supernatants of spleen cells (from a) activated with anti-CD3 antibodies and anti-CD28 antibodies. (e) The serum level of EV-specific immunoglobulin (Ig) $\mathrm{G}_{1}(P<0.05$ refers to a significant difference compared with the PBS treatment). 'Bacteria P.O.' and 'EV P.O.' refer to the oral administration of $H$. pylori and H. pylori-derived EVs, respectively. 'Bacteria I.P.' and 'EV I.P.' refer to the intraperitoneal administration of $H$. pylori and $H$. pylori-derived EVs, respectively. 

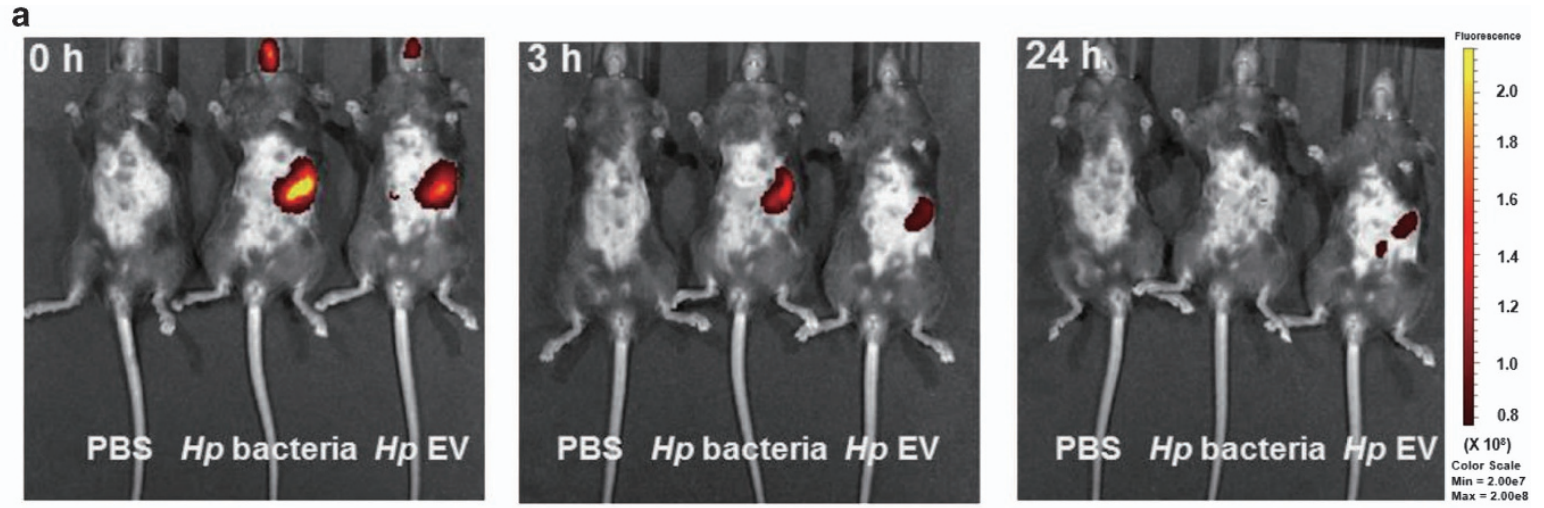

b

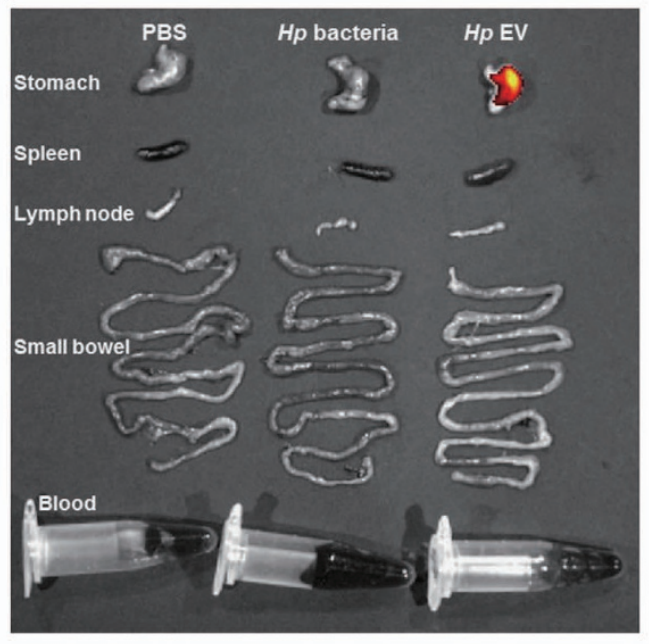

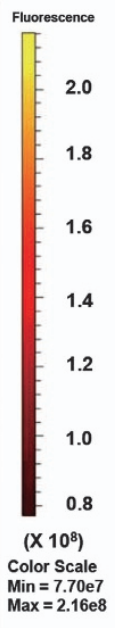

$\operatorname{ax}=2.16 \mathrm{e} 8$

\section{C}
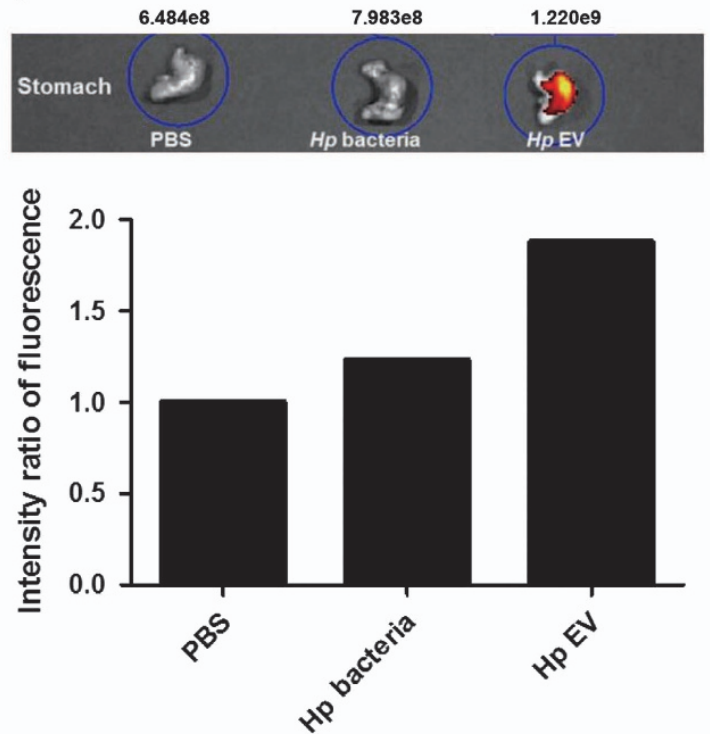

Dil
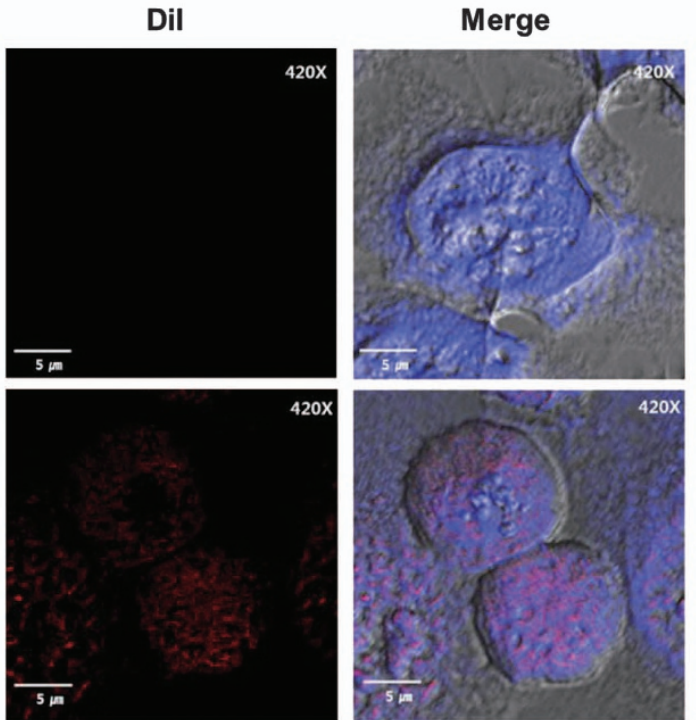

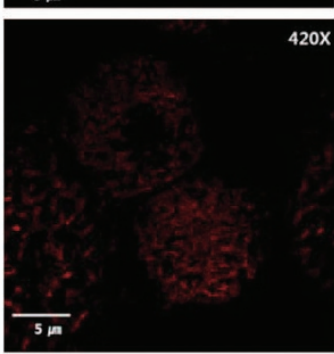

d

PBS
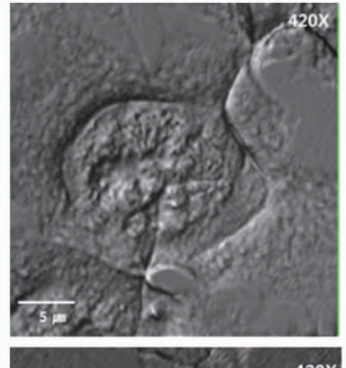

DAPI
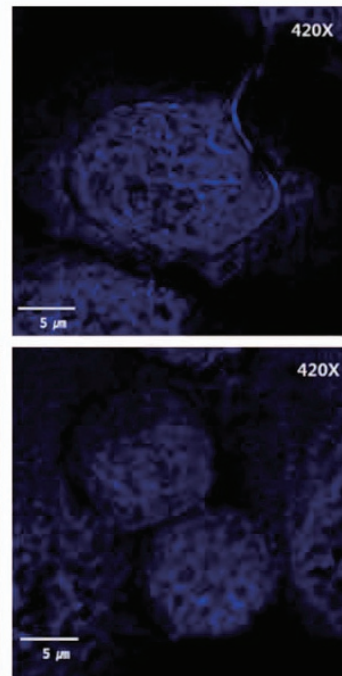

Figure 6 Infiltration of $\mathrm{H}$. pylori-derived Extracellular vesicles (EVs) into the gastric mucosal layer of C57BL/6 mice and endocytosis of EVs into the stomach epithelial cells. (a) Fluorescent imaging of mice at 0,3 and $24 \mathrm{~h}$ post oral administration of Cy7-labeled $\mathrm{H}$. pylori and H. pylori-derived EVs. (b) Fluorescence imaging of dissected organs $24 \mathrm{~h}$ post oral administration of PBS, Cy7-labeled H. pylori, and Cy7-labeled H. pylori-derived EVs. (c) Quantitation of fluorescent intensities in the stomachs of mice that received PBS, $H$. pylori (Bacteria), and Cy7-labeled H. pylori-derived EVs (EV). (d) Endocytosis of EVs into the stomach epithelial cells. Fluorescent images of human stomach epithelial cells (blue, DAPI) treated with Dil-labeled EVs (red) were obtained using confocal microscopy. 


\section{Endocytosis of EVs into stomach epithelial cells}

We evaluated the endocytosis of $H$. pylori-derived EVs into target cells. AGS cells were exposed to DiI-stained cell-free EVs. Large amounts of DiI-stained cell-free EVs were observed inside the AGS cells within the DAPI-stained areas $12 \mathrm{~h}$ post injection (Figure 6d). However, the PBS treatment did not show any EV-like particles within or outside the cells. DiI-stained $H$. pylori-derived EVs readily entered AGS cells and were easily distinguished from PBS-treated controls ('EV' and 'PBS,' respectively, Figure 6d). In total, our results imply that EVs can enter the stomach cells of mice via oral routes and can remain inside the cells for an extended time.

\section{DISCUSSION}

Almost all of the studies of microbiota in the human stomach have been based on tissue or media from cultured gastric juices. However, in the present study, we analyzed gastric juices directly using genome sequencing. Gastric cancer patients have a greater variety of bacteria than those with gastritis. We speculated that the inflammatory process suppresses acid production. ${ }^{30-32}$ In recent reports, gastric cancer patients showed an increased level of Streptococcus but a decreased level of Helicobacter according to biopsy. ${ }^{4,22,23}$ We analyzed sequencing results by separately studying bacteria and EVs collected from gastric juice. Our data showed significant increases in Streptococcus and Helicobacter in gastric cancer patients, which is probably due to the differences in the biopsy and gastric juice samples. We assumed that biopsy only represented part of the stomach, whereas gastric juice represents most of the stomach. There was no correlation between Helicobacter and Streptococcus in the bacterial pellets or EVs. However, Helicobacter in the bacterial pellets and EVs from gastric juices were correlated. Thus, we studied H. pylori-derived EVs (Supplementary Figure 3).

EVs are transporters of DNA, RNA, phospholipid and proteins in all forms of life $\mathrm{e}^{9,33}$ including $H$. pylori. ${ }^{34}$ In particular, H. pylori-derived EVs and their interaction with human cells have been extensively characterized, demonstrating that EVs contain more than 1500 different proteins including adhesion- and virulence-associated proteins. ${ }^{16}$ Internalization of $H$. pylori-derived EVs into AGS cells has also been well characterized. The binding of EVs to AGS cells is enhanced by the presence of VacA cytotoxin, whereas VacA-containing vesicle uptake is inhibited by LPS, and VacA-depleted EVs are dependent on clathrin-mediated endocytosis for uptake into AGS cells. ${ }^{17}$ In the present study, we also showed that H. pylori-derived EVs are taken up by AGS cells, and EVs can even infiltrate the gastric mucosa of the stomach and remain there for up to $24 \mathrm{~h}$. In contrast, Cy7-labeled H. pylori was not present in vivo in this amount of time. Although EV derived gut microbes, such as Pseudomonas panacis, penetrate the gut barrier and travel throughout the entire body, ${ }^{35}$ H. pylori-derived EVs remained in the stomach. Together with the data showing that EVs can induce cytokine production from gastric epithelial cells, these results suggest that $H$. pylori-derived EVs appear to be the main mediator of $H$. pylori-derived gastric malignancy.

The infection of gastric epithelial cells (AGS) by $H$. pylori results in the overexpression of IL- 8 and MCP-1 with Ras, MAPK cascade, AP-1 and NF- $\kappa \mathrm{B}$ acting upstream of the transcriptional pathways of these chemokines. ${ }^{36}$ The upregulation of pro-inflammatory cytokines IL-1, IL-6 and TNF- $\alpha$ has also been reported. ${ }^{37}$ Cis, trans-isomerase secreted by $H$. pylori is able to drive a $\mathrm{TH}_{17}$ response. $H$. pylori infection induced low cytotoxic gastric tumor-infiltrating lymphocytes response, matrix degradation and pro-angiogenic pathways are related to the pathogenesis of gastric cancer. ${ }^{38}$ Of the various pathogenic factors derived from $H$. pylori, CagA is one of the major agents leading to gastric cancer; among the patients infected with $H$. pylori, those who had IgG antibodies against CagA had a higher chance of developing gastric cancer. ${ }^{39}$ In addition, VacA is also an agent that induces acute inflammation of the gastric mucosa and plays a role in vacuolization in many cells. ${ }^{40}$ There are many reports on H. pylori-derived EVs. VacA-positive H. pylori-derived EVs have been reported to induce apoptosis in AGS and increase the carcinogenic potential in the stomach. ${ }^{14,15}$ Recently, CagA was detected in serum EVs from patients infected with H. pylori, and it has been reported that CagA-containing EVs can be taken up by AGS cells and induce morphological changes. ${ }^{41}$ CagA is localized at the surface, in contrast to VacA, which is located at both the surface and inner membrane of $\mathrm{H}$. pylori-derived EVs. ${ }^{16}$ Combined with the previous aforementioned data supporting the characteristics and mechanisms of $H$. pylori-derived EVs and their interactions with host human cells, our current data provide the first direct links between $H$. pylori-derived EVs and their pathogenic contribution to the induction of host immune responses in vitro and in vivo. We showed that $H$. pylori-derived EVs could penetrate gastric cancer and macrophage cell lines and induce IL-1ß, IL-6, IL- 8 and TNF- $\alpha$ expression in vitro (Figures $6 \mathrm{a}$ and b; Supplementary Figures $4 \mathrm{a}$ and b). H. pylori-derived EVs also elicited $\mathrm{TH}_{1}$ - and $\mathrm{TH}_{17}$-mediated immune responses through IL-17 and IFN- $\gamma$ induction in vivo. These combined data clearly demonstrate that EVs independent of $H$. pylori cells can penetrate and induce immune responses in target cells. Our data indicate that both bacteria and EVs induce localized immune responses with oral administration and systemic immune responses with intraperitoneal administration. Based on our in vivo imaging data, we suggest that these effects are due to stomach-specific relationships.

As indirect evidence of the relationship between $H$. pylori-derived EVs and gastric cancer, a small-scale analysis showed that both $H$. pylori cells and $H$. pylori-derived EVs were present at a significantly higher level in the gastric juices of gastric cancer patients than in normal controls. Due to the lack of appropriate animal models in which $H$. pylori and H. pylori-derived EVs not only induced inflammatory cytokines, but ultimately resulted in gastric cancer in a reasonable time frame, we utilized the $16 \mathrm{~S}$ rDNA metagenome analysis of gastric juices from normal control and gastric cancer 
patients. In this analysis, not only the cells pelleted by centrifugation from gastric juices but also the cell-free EVs (Methods) were subjected to analysis of microbial composition. Both $H$. pylori cells and $H$. pylori-derived EVs were significantly more abundant in gastric cancer samples compared with normal controls. These results suggest that EVs secreted from Helicobacter in the stomach lead to infiltration of the gastric mucosae and eventually to gastric cancer. The sequencing data from the gastric juices demonstrate that EVs derived from other bacteria, such as Streptococcus, require additional study in the context of gastric disease.

In summary, the present study showed that $H$. pylori-derived EVs, which are abundant in the gastric juices of GC patients, can induce inflammation and possibly cancer in the stomach, mainly via the production of inflammatory mediators, such as IL-8, from gastric epithelial cells after selective uptake by the cells.

\section{CONFLICT OF INTEREST}

The authors declare no conflict of interest.

\section{ACKNOWLEDGEMENTS}

We thank the animal facility members of Pohang University of Science and Technology Biotech Center (Pohang University of Science and Technology, Pohang, Republic of Korea). This work was funded by the Ministry of Science, ICT, and Future Planning (NRF-2014R1A1A1005144 and NRF-2014K1B1A1073720), by the Basic Science Research Program funded by the Ministry of Education (NRF-2012R1A1A2007865) and by Korea Ministry of Health \&Welfare, Republic of Korea (HI16C0998).

Author contributions: HIC, BJK and JGK contributed to the study concept and design; HIC, MR and JGK contributed to acquisition of data; HIC, MR and JGK contributed to the analysis and interpretation of data; HIC, J-PC, JKH and JGK contributed to drafting of the manuscript; HIC and JGK contributed to critical revision of the manuscript for important intellectual content; HIC, JKH and MR contributed to the statistical analysis; MR and JGK funded the study; J-PC and JWS contributed to administrative, technical or material support; MR and JGK contributed to study supervision.

1 Ferreira RM, Machado JC, Figueiredo C. Clinical relevance of Helicobacter pylori vaca and caga genotypes in gastric carcinoma. Best Pract Res Clin Gastroenterol 2014; 28: 1003-1015.

2 Wroblewski LE, Peek RM, Wilson KT. Helicobacter pylori and gastric cancer: factors that modulate disease risk. Clin Microbiol Rev 2010; 23: 713-739.

3 Kurokawa K, Itoh T, Kuwahara T, Oshima K, Toh H, Toyoda A et al. Comparative metagenomics revealed commonly enriched gene sets in human gut microbiomes. DNA Res 2007; 14: 169-181.

4 Bik EM, Eckburg PB, Gill SR, Nelson KE, Purdom EA, Francois F et al. Molecular analysis of the bacterial microbiota in the human stomach. Proc Natl Acad Sci USA 2006; 103: 732-737.

5 Sommer F, Bäckhed F. The gut microbiota-masters of host development and physiology. Nat Rev Microbiol 2013; 11: 227-238.

6 Aviles-Jimenez F, Vazquez-Jimenez F, Medrano-Guzman R, Mantilla A, Torres J. Stomach microbiota composition varies between patients with non-atrophic gastritis and patients with intestinal type of gastric cancer. Sci Rep 2014; 4: 4202.

7 Tucci A, Bisceglia M, Rugge M, Tucci P, Marchegiani A, Papadopoli G et al. Clinical usefulness of gastric-juice analysis in 2007: the stone that the builders rejected has become the cornerstone. Gastrointest Endosc 2007 66: 881-890.

8 Yang $\mathrm{Y}$, Shao $\mathrm{Y}$, Zhu M, Li Q, Yang F, Lu X et al. Using gastric juice Incrna-abhd11-as1 as a novel type of biomarker in the screening of gastric cancer. Tumour Biol 2015; 37: 1-6.

$9 \mathrm{Kim} \mathrm{JH}$, Lee J, Park J, Gho YS. Gram-negative and gram-positive bacterial extracellular vesicles. Semin Cell Dev Biol 2015; 40: 97-104.

10 van der Pol E, Böing AN, Harrison P, Sturk A, Nieuwland R. Classification, functions, and clinical relevance of extracellular vesicles. Pharmacol Rev 2012; 64: 676-705.

11 Hong SW, Kim MR, Lee EY, Kim J, Kim YS, Jeon S et al. Extracellular vesicles derived from staphylococcus aureus induce atopic dermatitis-like skin inflammation. Allergy 2011; 66: 351-359.

12 Park K-S, Choi K-H, Kim Y-S, Hong BS, Kim OY, Kim JH et al. Outer membrane vesicles derived from Escherichia coli induce systemic inflammatory response syndrome. PLOS ONE 2010; 5: e11334.

13 Park K-S, Lee J, Jang SC, Kim SR, Jang MH, Lötvall J et al. Pulmonary inflammation induced by bacteria-free outer membrane vesicles from Pseudomonas aeruginosa. Am J Respir Cell Mol Biol 2013; 49: 637-645.

14 Ayala G, Torres L, Espinosa M, Fierros-Zarate G, Maldonado V, Meléndez-Zajgla J. External membrane vesicles from Helicobacter pylori induce apoptosis in gastric epithelial cells. FEMS Microbiol Lett 2006; 260: 178-185.

15 Chitcholtan K, Hampton MB, Keenan JI. Outer membrane vesicles enhance the carcinogenic potential of Helicobacter pylori. Carcinogenesis 2008; 29: 2400-2405.

16 Olofsson A, Vallström A, Petzold K, Tegtmeyer N, Schleucher J, Carlsson S et al. Biochemical and functional characterization of Helicobacter pylori vesicles. Mol Microbiol 2010; 77: 1539-1555.

17 Parker H, Chitcholtan K, Hampton MB, Keenan JI. Uptake of helicobacter pylori outer membrane vesicles by gastric epithelial cells. Infect Immun 2010; 78: 5054-5061.

18 Edgar RC. Search and clustering orders of magnitude faster than blast. Bioinformatics 2010; 26: 2460-2461.

19 Caporaso JG, Kuczynski J, Stombaugh J, Bittinger K, Bushman FD, Costello EK et al. Qiime allows analysis of high-throughput community sequencing data. Nat Methods 2010; 7: 335-336.

$20 \mathrm{Kim} \mathrm{H}$, Seo JY, Kim KH. Effects of mannitol and dimethylthiourea on Helicobacter pylori-induced il-8 production in gastric epithelial cells. Pharmacology 1999; 59: 201-211.

$21 \mathrm{Kim} \mathrm{H}$, Seo JY, Kim KH. Inhibition of lipid peroxidation, nf-kb activation and il-8 production by rebamipide in Helicobacter pylori-stimulated gastric epithelial cells. Dig Dis Sci 2000; 45: 621-628.

22 Abreu MT, Peek RM. Gastrointestinal malignancy and the microbiome. Gastroenterology 2014; 146: 1534-1546. e1533.

23 Khosravi $\mathrm{Y}$, Dieye Y, Poh BH, Ng CG, Loke MF, Goh KL et al. Culturable bacterial microbiota of the stomach of Helicobacter pylori positive and negative gastric disease patients. SCI World J 2014; 2014: 610421.

24 Peek RM, Fiske C, Wilson KT. Role of innate immunity in Helicobacter pylori-induced gastric malignancy. Physiol Rev 2010; 90: 831-858.

25 Obonyo M, Sabet M, Cole SP, Ebmeyer J, Uematsu S, Akira S et al. Deficiencies of myeloid differentiation factor 88 , toll-like receptor 2(tIr2), or tir4 produce specific defects in macrophage cytokine secretion induced by Helicobacter pylori. Infect Immun 2007; 75: 2408-2414.

26 Suganuma M, Kuzuhara T, Yamaguchi K, Fujiki H. Carcinogenic role of tumor necrosis factor- $\alpha$ inducing protein of Helicobacter pylori in human stomach. BMB Rep 2006; 39: 1-8.

27 Kim G-Y, Lee J-W, Ryu H-C, Wei J-D, Seong C-M, Kim J-H. Proinflammatory cytokine il-1 $\beta$ stimulates il-8 synthesis in mast cells via a leukotriene b4 receptor 2-linked pathway, contributing to angiogenesis. J Immunol 2010; 184: 3946-3954.

28 Su B, Ceponis PJ, Lebel S, Huynh H, Sherman PM. Helicobacter pylori activates toll-like receptor 4 expression in gastrointestinal epithelial cells. Infect Immun 2003; 71: 3496-3502.

29 Jang SC, Kim SR, Yoon YJ, Park KS, Kim JH, Lee J et al. In vivo kinetic biodistribution of nano-sized outer membrane vesicles derived from bacteria. Small 2015; 11: 456-461.

30 Blaser MJ, Atherton JC. Helicobacter pylori persistence: biology and disease. J Clin Invest 2004; 113: 321.

31 McColl K, El-Omar E, Gillen D. Interactions between H. pylori infection, gastric acid secretion and anti-secretory therapy. Br Med Bull 1998; 54: 121-138. 
32 Suerbaum S, Michetti P. Helicobacter pylori infection. N Engl J Med 2002; 347: 1175-1186.

33 Coakley G, Maizels RM, Buck AH. Exosomes and other extracellular vesicles: the new communicators in parasite infections. Trends Parasitol 2015; 31: 477-489.

34 Fiocca R, Necchi V, Sommi P, Ricci V, Telford J, Cover TL et al. Release of Helicobacter pylori vacuolating cytotoxin by both a specific secretion pathway and budding of outer membrane vesicles. Uptake of released toxin and vesicles by gastric epithelium. J Pathol 1999; 188: 220-226.

35 Choi Y, Kwon Y, Kim D-K, Jeon J, Jang SC, Wang T et al. Gut microbederived extracellular vesicles induce insulin resistance, thereby impairing glucose metabolism in skeletal muscle. Sci Rep 2015; 5: 15878.

$36 \mathrm{Seo} \mathrm{JH}, \mathrm{Lim}$ JW, Kim H, Kim KH. Helicobacter pylori in a korean isolate activates mitogen-activated protein kinases, ap-1, and nf-kb and induces chemokine expression in gastric epithelial ags cells. Lab Invest 2004; 84: 49-62.

37 Yamaoka Y, Kita M, Kodama T, Sawai N, Kashima K, Imanishi J. Induction of various cytokines and development of severe mucosal inflammation by caga gene positive Helicobacter pylori strains. Gut 1997; 41: 442-451.

38 Amedei A, Munari F, Della Bella C, Niccolai E, Benagiano M, Bencini L et al. Helicobacter pylori secreted peptidyl prolyl cis, trans-isomerase drives th17 inflammation in gastric adenocarcinoma. Intern Emerg Med 2014; 9: 303-309.
39 Parsonnet J, Friedman G, Orentreich N, Vogelman H. Risk for gastric cancer in people with caga positive or caga negative Helicobacter pylori infection. Gut 1997; 40: 297-301.

40 Supajatura V, Ushio H, Wada A, Yahiro K, Okumura K, Ogawa H et al. Cutting edge: Vaca, a vacuolating cytotoxin of Helicobacter pylori, directly activates mast cells for migration and production of proinflammatory cytokines. J Immunol 2002; 168: 2603-2607.

41 Shimoda A, Ueda K, Nishiumi S, Murata-Kamiya N, Mukai S-a, Sawada S-i et al. Exosomes as nanocarriers for systemic delivery of the Helicobacter pylori virulence factor caga. Sci Rep 2016; 6: 18346.

$($ (1) $\Theta \Theta$ This work is licensed under a Creative Commons Attribution-NonCommercial-NoDerivs 4.0 International License. The images or other third party material in this article are included in the article's Creative Commons license, unless indicated otherwise in the credit line; if the material is not included under the Creative Commons license, users will need to obtain permission from the license holder to reproduce the material. To view a copy of this license, visit http://creativecommons.org/licenses/by-nc-nd/4.0/

Supplementary Information accompanies the paper on Experimental \& Molecular Medicine website (http://www.nature.com/emm) 